\title{
Interleukin- 6 blockade and cytokine release syndrome in coronavirus disease of 2019: Is that a lot? Thoughts from the emergency department
}

\section{Sadaf Sheikh*}

Emergency Medicine Department, Sultan Qaboos University Hospital, Muscat, Oman

\section{Dear Editor,}

Cytokine storm remains the main feature in pathophysiology of the coronavirus disease 2019 (COVID-19) causing adverse effects from interleukin (IL) $1 \beta, 6$, and tumor necrosis factor- $\alpha$. We are sharing some insights over the role of IL- 6 driven immune dysregulation with anti-cytokine therapies targeting IL-1, 6, and tumor necrosis factor inhibitors $(1,2)$. We believe that it is crucial for emergency physicians to be aware of the initial evaluation of IL-6 levels for patients with coronavirus disease due to its potential benefits and its supportive role in assessing worsening features and disease progression.

Coronaviruses with an acute hyper-inflammatory syndrome can activate dysregulated host immune responses. It initiates cytokine release syndrome with clinical features such as fever, hypoxia and raised inflammatory markers. The key factor related to this syndrome is the increased production of IL- 6 by the infected T cells, macrophages, and dendritic cells (1). Viral load with raised IL-6 levels in critically ill patients infected with coronavirus disease is worth observing. IL-6 had been under consideration for viral epidemics in the past and many reports have found high cytokine levels in such patients (1-3).

IL-6 is a pivotal pro-inflammatory cytokine with a crucial role in inflammation and infection. It has also attracted high levels of interest. The antibodies that block the IL-6 receptor are currently under clinical trials for the potential treatment of COVID-19. It is believed that inflammation is an important causative factor of lung damage in coronavirus disease of 2019. Observational data suggest employing IL-6 inhibition to suppress tissue injury. Experimental studies show that elevated IL-6 is found in severe infection, hence cytokine storm blockers and immune host modulators are used to deal with overwhelming inflammation (2-5). Cytokine storm with the release of IL-6, 1, 12, 18, and tumor necrosis factor-alpha were observed in the severe cases of COVID-19 infections. These inflammatory mediators cause pulmonary inflammatory response with increased alveolar-capillary gas exchange causing oxygenation difficult in these patients. Hypoxia and the profound inflammatory response observed in COVID-19 pneumonia encouraged scientists to use IL- 6 or receptorblocking antibodies. Agents such as tocilizumab, sarilumab, and siltuximab have been used in clinical trials in severe cases of COVID-19 pneumonia (3-5),

Tocilizumab is a recombinant humanized monoclonal antibody that deals with soluble and membranebound IL-6 receptor with complete signaling blockade resulting in a rapid recovery from fever and respiratory distress (3-6). This agent shows efficacy in the treatment of rheumatoid arthritis. It is proposed as a potential treatment option for COVID-19 with a rationale to control excessive inflammation. A non-peer reviewed study by Xu et al was conducted on 21 patients with severe symptoms of coronavirus disease. In this study, Lopinavir and Methylprednisolone with $400 \mathrm{mg}$ of Tocilizumab single dose were given to patients $(3,4)$. Improvement with low requirement for oxygen, respiratory symptoms, and normalizing lymphocyte counts were observed and $(3,4)$ no records of any adverse events or deaths were seen (3).

Not all patients respond to IL- 6 inhibition and this could be explained by neutralizing antibodies that develop

Received: 20 February 2021 Accepted: 25 May 2021 Published online: 5 June 2021

*Corresponding author: Sadaf Sheikh, Sultan Qaboos University Hospital, Muscat, Email: Oman sheikh.sadaf@gmail.com

Competing interests: None.

Funding information: None.

Citation: Sheikh S. Interleukin-6 blockade and cytokine release syndrome in coronavirus disease of 2019: Is that a lot? Thoughts from the emergency department. Journal of Emergency Practice and Trauma 2021; 7(2): 80-81. doi: 10.34172/jept.2021.21. 
against the blocking agent. Increased susceptibility to bacterial infections is another pressing concern due to impaired acute-phase response caused by the IL- 6 blockade. A recent study undertaken on the patients infected with coronavirus disease in the intensive care unit showed that the use of Tocilizumab was linked with a high incidence of secondary bacterial infections (3). It is also associated with higher rates of hospital-acquired pneumonia and ventilator-associated pneumonia (3-6). These findings cast a shadow over the idea of taking IL inhibition as an agent helping the immune host response to fight against severe infection like COVID-19.

Elevated IL-6 levels have been reported in patients with other immune and rheumatologic conditions and there is no evidence concerning the raised IL- 6 in patients with autoimmune pathologies and its association with acute lung injury. IL-6 levels in COVID-19can be elevated beyond normal limits and no study involving the use of IL-6 in humans indicates lung injury or other organ malfunction making it difficult to attribute to IL-6 biological actions (3-6). As data is spreading expeditiously with numerous agents such as hydroxychloroquine, azithromycin, nitazoxanide, lopinavir-ritonavir, remdesivir, corticosteroids, and tocilizumab, emergency physicians and other clinicians should take caution using off label medications with questionable efficacy. IL-6 inhibitors such as tocilizumab can be considered as topline treatment options keeping its pro-inflammatory effect for COVID-19. It might be a sound strategy to treat the infection like what was done in China and Italy. Those well-intended treatment options require credible randomized controlled trials.

It is imperative that emergency physicians and other clinicians evaluate a cytokine panel to identify the needs of each patient before the administration of selective immunosuppressive therapy. A combination of antiviral and immunosuppressive therapies that decrease the viral load should be considered for future treatment options.

Ethical issue

Not applicable

\section{References}

1. Huang C, Wang Y, Li X, Ren L, Zhao J, Hu Y, et al. Clinical features of patients infected with 2019 novel coronavirus in Wuhan, China. Lancet 2020; 395(10223): 497-506. doi: 10.1016/s0140-6736(20)30183-5.

2. Zhang C, Wu Z, Li JW, Zhao H, Wang GQ. Cytokine release syndrome in severe COVID-19: interleukin-6 receptor antagonist tocilizumab may be the key to reduce mortality. Int J Antimicrob Agents 2020; 55(5): 105954. doi: 10.1016/j. ijantimicag.2020.105954.

3. Zumla A, Hui DS, Azhar EI, Memish ZA, Maeurer M. Reducing mortality from 2019-nCoV: host-directed therapies should be an option. Lancet 2020; 395(10224): e35-e6. doi: 10.1016/s0140-6736(20)30305-6.

4. Zhou Y, Fu B, Zheng X, Wang D, Zhao C, Qi Y, et al. Pathogenic $\mathrm{T}$ cells and inflammatory monocytes incite inflammatory storm in severe COVID-19 patients. Natl Sci Rev 2020; 7(6): 998-1002. doi: 10.1093/nsr/nwaa041

5. Gong J, Dong H, Xia Q, Huang Z, Wang D, Zhao Y, et al. Correlation analysis between disease severity and inflammation-related parameters in patients with COVID-19 pneumonia. medRxiv 2020. doi: 10.1101/2020.02.25.20025643.

6. Rahmati M, Moosavi MA. Cytokine-targeted therapy in severely ill COVID-19 patients: options and cautions. Eurasian J Med Oncol 2020; 4(2): 179-81. doi: 10.14744/ ejmo.2020.72142. 